includes many corrections not given by Schering in his appendix to Gauss's volume, or by Perott.

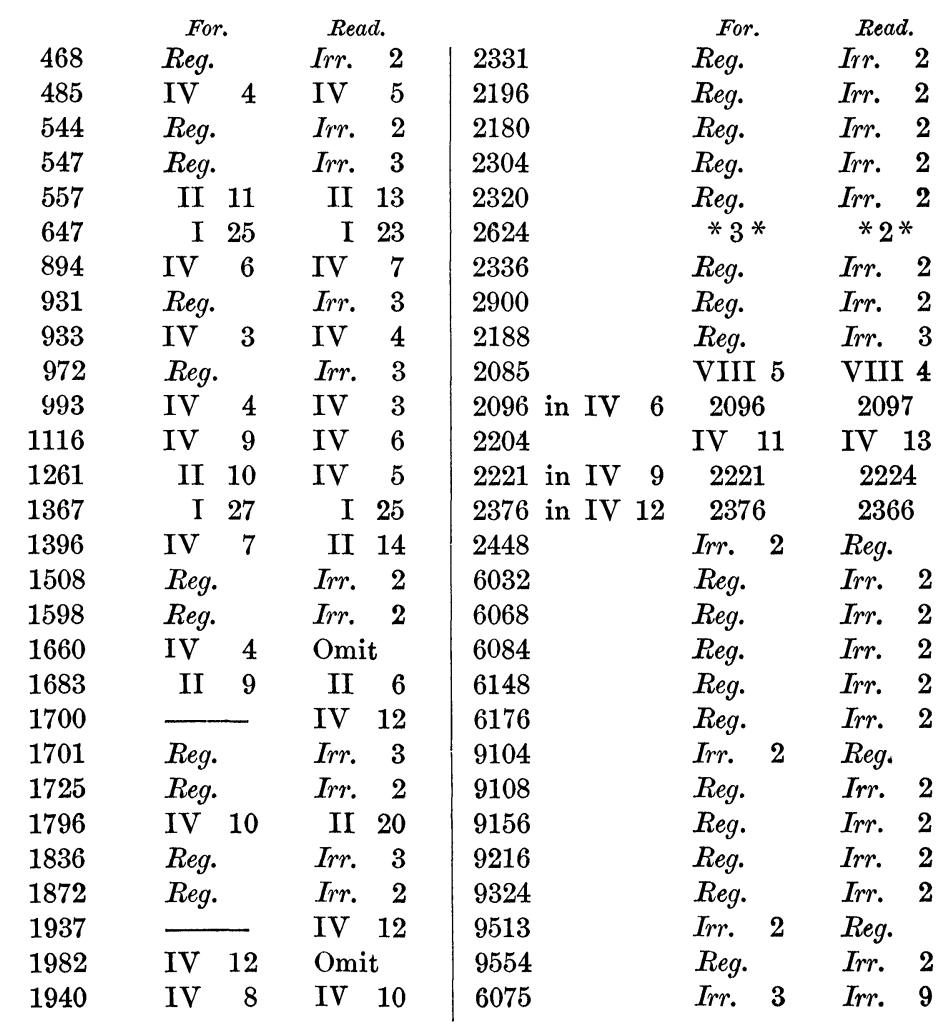

\title{
THE LOGARITHM AS A DIRECT FUNCTION.
}

BY DR. EMORY MCCLINTOCK.

(Read before the American Mathematical Society, February 28, 1903.)

IN a paper of the same title published in the Annals of Mathematics for January, 1903, Mr. J. W. Bradshaw defines $\log x$ as a direct function of $x$, namely,

$$
\log x=\int_{1}^{x} x^{-1} d x
$$


Attention being thus drawn to the subject, I think the time opportune to repeat and amplify a proposition of my own for the same general purpose.

In 1879 (American Journal of Mathematics, II, 101, etc.) I spoke of "the difficulty of comprehending logarithms," and quoted De Morgan's dictum that "the only definition of $\log x$ used in analysis is $y$, where $e^{y}=x . "$ After discussing this definition I said, "Another and, when duly weighed, most satisfactory definition may be derived from any one of an unlimited number of vanishing fractions, special cases of the general form $\log x=h^{-1}\left(x^{(1-a) h}-x^{-a h}\right)$, where $h$ is infinitely reduced. *** This fraction is doubtless novel, though one case of it, where $a=0$, is known. Even that case has not, I presume, been suggested heretofore as a definition. $* * *$ The various theorems pertaining to logarithms may be derived with the utmost facility by the aid of these vanishing-fraction definitions. Thus, if $\alpha=0$, we have by expansion

$$
\log (1+x)=\frac{(1+x)^{h}-1}{h}[h=0]=x-\frac{1}{2} x^{2}+\frac{1}{3} x^{3}-\cdots,
$$

To develop this proposition more fully, let us consider the function $y=h^{-1}\left(x^{h}-1\right)$. Let $k$ be positive, and, first, let $h=k$. When $x=1, y=0$; when $x=\infty, y=\infty$; and as $x$ increases continuously from 1 towards $\infty$, there is one and only one corresponding value of $y$, which increases accordingly from 0 towards $\infty$. Secondly, let $h=-k$. Here again, when $x=1, y=0$; and, in the function $y=k^{-1}\left(1-x^{-k}\right)$, as $x$ increases continuously from 1 towards $\infty$, there is one and only one corresponding value of $y$, which increases accordingly from 0 to $k^{-1}$, a limit which tends towards $\infty$ if $k$ tends towards 0 . When $h=-k, y=k^{-1}\left(x^{k}-1\right) x^{-k}$, which differs only by the factor $x^{-k}$ from the value of $y$ when $h=k$. The smaller $k$ is taken, the nearer this factor is to 1 , so that the limit of the value of $y$, for $h=0$, is the same whether $h$ is positive or negative, while

$$
y_{[h<0]}<\lim _{h=0} y<y_{[h>0]} .
$$

The limit is therefore a 1 to 1 function of $x>1$. When $x=1$, the limit is 0 . When $0<x<1$, let $x=u^{-1}$, where $u>1$; then we have $h^{-1}\left(x^{h}-1\right)=h^{-1}\left(1-u^{h}\right) x^{h}$, and, since $\lim _{h=0} x^{h}=1$, $\lim _{h=0} h^{-1}\left(x^{h}-1\right)=-\lim _{h=0} h^{-1}\left(u^{h}-1\right)$. 
Let us define the logarithm of $x$ (positive) as $\lim _{h=0} h^{-1}\left(x^{h}-1\right.$ ), and denote it by $\log x$. We have just found that $\log \left(x^{-1}\right)$ $=-\log x$. Since $\lim _{h=0} b^{h}=1$,

$$
\log a=\lim _{h=0} h^{-1}\left(a^{h} b^{h}-b^{h}\right)=\log (a b)-\log b .
$$

This is the chief property of logarithms. Hence, $\log \left(a^{2}\right)=$ $2 \log a, \log \left(a^{n}\right)=n \log a$, and if $b=a^{n}, \operatorname{lo}\left(b^{1 / n}\right)=1 / n \log b$, which might be used, as by Mr. Bradshaw from another definition of $\log x$, to show that for every positive number $b$ there exists one and only one positive $n$th root. Here $n$ is a whole number. It follows that $\log b^{m / n}=m \log \left(b^{1 / n}\right)=m / n \log b$. If we take $n$ incommensurable, let $a=b^{m}$, where $m$ is an integer, $b=1+c$, and $-1<c<1$. Employing the binomial expansion,

$$
\begin{aligned}
\log \left(b^{n}\right)=\lim _{h=0} h^{-1}\left[(1+c)^{n h}-1\right] & =n\left(c-\frac{1}{2} c^{2}+\frac{1}{3} c^{3}-\cdots\right) \\
& =n \log (1+c)=n \log b .
\end{aligned}
$$

Hence

$$
\log \left(a^{n}\right)=\log \left(b^{m n}\right)=m \log \left(b^{n}\right)=n m \log b=n \log a .
$$

That the continuous function $\log x$ has a continuous derivative $x^{-1}$ may be shown thus, with $\Delta x<x$ :

$$
\frac{d \log x}{d x}=\lim _{\Delta x=0} \lim _{h=0} h^{-1}(\Delta x)^{-1}\left[(x+\Delta x)^{h}-x^{h}\right] .
$$

If we expand the part within the brackets and divide the resulting series throughout by $h \Delta x$, we have

$$
\begin{aligned}
& \frac{d \log x}{d x}=\lim _{\Delta x=0} \lim _{h=0}\left[x^{h-1}\right.+\frac{1}{2}(h-1)(\Delta x) x^{h-2}+ \\
&\left.\frac{1}{2 \cdot 3}(h-1)(h-2)(\Delta x)^{2} x^{h-3}+\cdots\right] .
\end{aligned}
$$

If we first put $h=0$, the part within brackets becomes $x^{-1}$ $-\frac{1}{2}(\Delta x) x^{-2}+\frac{1}{3}(\Delta x)^{2} x^{-3}-\cdots$, which is $x^{-1}$ when $\Delta x=0$. If we first put $\Delta x=0$, the part within brackets becomes $x^{h-1}$, which is $x^{-1}$ when $h=0$. 